\title{
K-EUSO: An improved optical system for KLYPVE ultra-high energy cosmic ray space telescope
}

\author{
Fumiyoshi Kajino $^{* a}$, Toshikazu Ebisuzaki ${ }^{b}$, Marco Casolino $^{b}$, Yoshiyuki Takizawa $^{b}$, \\ Yoshiya Kawasaki $^{b}$, Naoto Sakaki ${ }^{c}$, Sergei Sharakin ${ }^{d}$, Pavel Klimov ${ }^{d}$, Mikhail I. \\ Panashyuk $^{d}$ for the JEM-EUSO Collaboration \\ ${ }^{a}$ Konan University, Okamoto 8-9-1, Higashinada, Kobe, Hyogo, 658-8501, Japan \\ ${ }^{b}$ RIKEN, Hirosawa 2-1, Wako, Saitama, 351-0198 Japan \\ ${ }^{c}$ Graduate School of Science, Osaka City University, Sugimoto 3-3-138, Sumiyoshi, Osaka \\ 558-8585, Japan \\ ${ }^{d}$ D.V. Skobeltsyn Institute of Nuclear Physics, M.V. Lomonosov Moscow State University, \\ Moscow 119991, Russia \\ E-mail: kajino@konan-u.ac.jp, ebisu@riken.jp, takky@riken.jp
}

\begin{abstract}
KLYPVE is a Russian science mission to detect ultra-high energy cosmic rays (UHECRs) above $5 \times 10^{19} \mathrm{eV}$. It will be attached to the Russian MRM-1 module onboard the International Space Station. The K-EUSO project is a result of the joint efforts of the JEM-EUSO collaboration to improve performance of the KLYPVE mission by employing the technologies (a corrective Fresnel lens, the focal surface detector, and a laser head) developed for the JEM-EUSO mission. The baseline design of the new KLYPVE uses a 3.4 m primary mirror, allowing large photon collection efficiency. Light from the mirror is focused by a $1.7 \mathrm{~m}$ diameter, double sided Fresnel lens above the focal surface. The lens enlarges the field of view to $\pm 14^{\circ}$ and reduces aberrations thus improving the effective area by more than a factor 7. The corrective lens improves the RMS spot size to about $5 \mathrm{~mm}$ by using the EUSO detector technology. This paper describes details of the baseline optical system for the K (KLYPVE)-EUSO.
\end{abstract}

The 34th International Cosmic Ray Conference,

30 July- 6 August, 2015

The Hague, The Netherlands

\footnotetext{
* Speaker.
} 


\section{Introduction}

The largest two ultra-high energy cosmic ray (UHECR) observatories in operation at present are the Pierre Auger Observatory, covering 3,000 $\mathrm{km}^{2}$ in the Southern Hemisphere (Mendoza, Argentina), and the Telescope Array (TA), covering $700 \mathrm{~km}^{2}$ in the Northern Hemisphere (Utah, USA). The current observatories are, although extremely large, not large enough to study UHECRs with the statistics necessary to unveil their sources. An important step towards the identification of UHECR sources is the measurement of anisotropies in the UHECR arrival directions. In fact, TA experiments suggested the presence of a hot spot a confidence level of $5.1 \sigma$ in their arrival direction map in the northern hemisphere[1]. In addition, a lower amplitude dipole anisotropy was reported by Auger, while the test for anisotropies based on correlations with active galactic nuclei continues to show a departure from isotropy at the $3 \sigma$ level but with a large background of isotropic events[2,3]. Space-based UHECR missions which can verify suggested anisotropies by the ground based experiments with a different instrument from space with a full sky coverage well suited to measure large-scale anisotropies. Space-based missions should be required to observe lager exposure than Auger above $5 \times 10^{19} \mathrm{eV}$.

KLYPVE (Kosmiceskie Luchi Predel' no Vysokikh Energii - Cosmic Rays of Ultra High Energy)[7] is a Russian science mission to detect UHECR above $5 \times 10^{19} \mathrm{eV}$. It will be attached to the Russian MRM-1 module onboard the International Space Station (ISS). The KLYPVE telescope is the latest in a series of detectors (TATIANA- 1 and -2 and the TUS instruments $[4,5,6,7]$ on board free-flyer satellites) of growing size and complexity built and launched in Russia to study UHECR. KLYPVE optics is the prime focus system using a $3.6 \mathrm{~m}$ primary mirror and a $1.2 \mathrm{~m}$ focal surface. The field of view and energy threshold of KLYPVE make the achievable performance unsatisfactory for extending the knowledge about UHECRs. Its expected exposure as a function of the energy is 0.5 times that of Auger. The KLYPVE team (Moscow State University) and the JEM-EUSO collaboration have studied how to improve the performance of the KLYPVE mission. The K(KLYPVE)-EUSO project is a result of these joint efforts. The K-EUSO telescope, using the technologies developed for the JEM-EUSO mission[8] (a corrective Fresnel lens, the focal surface detector, and a laser system), improves the effective area by more than a factor 7 from the original KLYPVE. The concept of the K-EUSO is shown in Fig. 1. Japanese team joins to KLYPVE mission in the frame work of the international JEM-EUSO collaboration to improve the optical design and to provide the corrective Fresnel lens, multi-anode photo-multiplier tubes (MAPMTs) to cover the focal surface and a laser head to monitor the atmospheric conditions. This paper describes the baseline optical system design and a Fresnel corrective lens to correct aberrations and enlarge the field of view, which are a part of the Japanese contributions to K-EUSO.

\section{The K-EUSO telescope}

The K-EUSO telescope reduced the energy threshold of the cosmic rays to $3 \times 10^{19} \mathrm{eV}$ and extended to $10^{20} \mathrm{eV}$. The use of a corrective Fresnel lens allows for a much larger field of view $\left( \pm 14^{\circ}\right)$ than did the original KLYPVE design $\left( \pm 7.5^{\circ}\right)$, while reducing the coma aberration and achieving smaller spot size the JEM-EUSO photo detector module enables K-EUSO to take full advantage of this smaller spot size. Main parameters of the K-EUSO telescope are summarized 


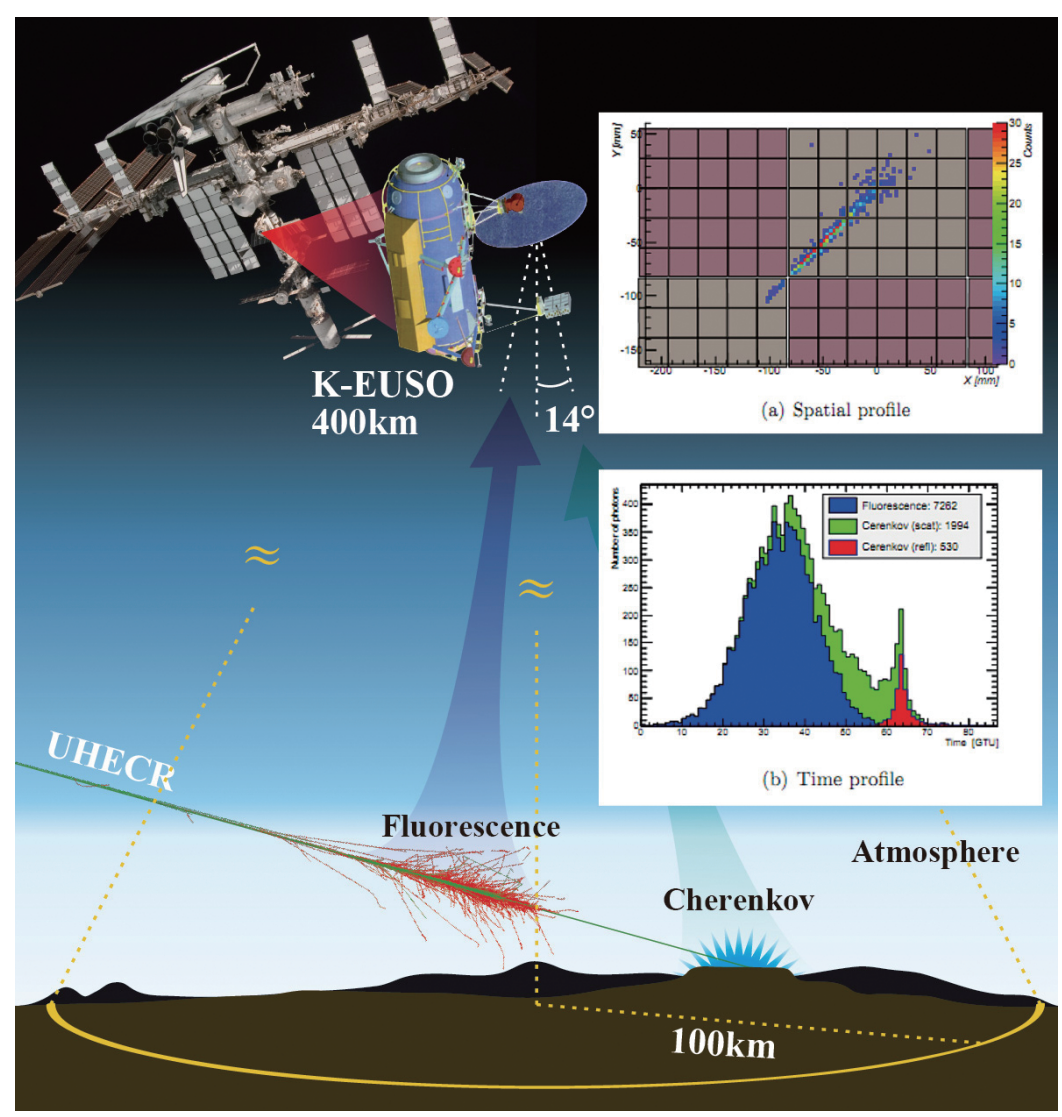

Figure 1: The concept of the K-EUSO to observe an UHECR from space. The UV light emitted by an extensive air shower is detected by K-EUSO . From the temporal and spatial profile of the shower the energy and arrival direction of the primary particle is reconstructed. Upper Right: The time and spatial resolution of an extensive air-shower with $10^{20} \mathrm{eV}$ and a zenith angle of $60^{\circ}$. A square represents MAPMTs with 64 channels, arranged in units of $6 \times 6$ photo-tubes called photon detection modules (PDMs). The small squares show the number of photons detected by each pixel. This event starts from the lower-left corner continues to the right top of the PDM. Lower Right: Time profile of photons, collected during $2.5 \mu$ s intervals. The signal has three components (UV light -blue, scattered Cherenkov - green, reflected Cherenkov - red).

in Table 1. K-EUSO will be launched with the Progress cargo and assembled by Extra-VehicularActivity on the MRM-1 module of the Russian section of the ISS, looking nadir. K-EUSO will be measuring the UV (300 - $430 \mathrm{~nm}$ ) light coming from EAS induced by UHECR interactions in the Earth's night atmosphere. The UV light is from fluorescence and Cherenkov radiation in the atmosphere. The UV light is focused through a primary mirror and a corrective Fresnel lens. The photons are detected by MAPMTs providing 119,808 pixels on the focal plane and are readout by electronics which records the track of the EAS with a time resolution of $2.5 \mu \mathrm{s}$. The angular resolution per pixel is $0.058^{\circ}$, corresponding to a pixel size of about $400 \mathrm{~m}$ assuming ISS altitude of $400 \mathrm{~km}$. The spatial and temporal profile of the shower will allow determination of the energy and direction of the primary particles with an accuracy better than a few degrees. Fig. 2 shows also a simulation of the time profile of the UV photons of the fluorescence and the Cherenkov origin as observed from K-EUSO. The telescope will point toward the nadir, observing a circular area of 
about $200 \mathrm{~km}$ in diameter, with a instantaneous geometrical area greater than $3.1 \times 10^{4} \mathrm{~km}^{2}$. The characteristics of K-EUSO compared with the original KLYPVE are shown in Table 2.

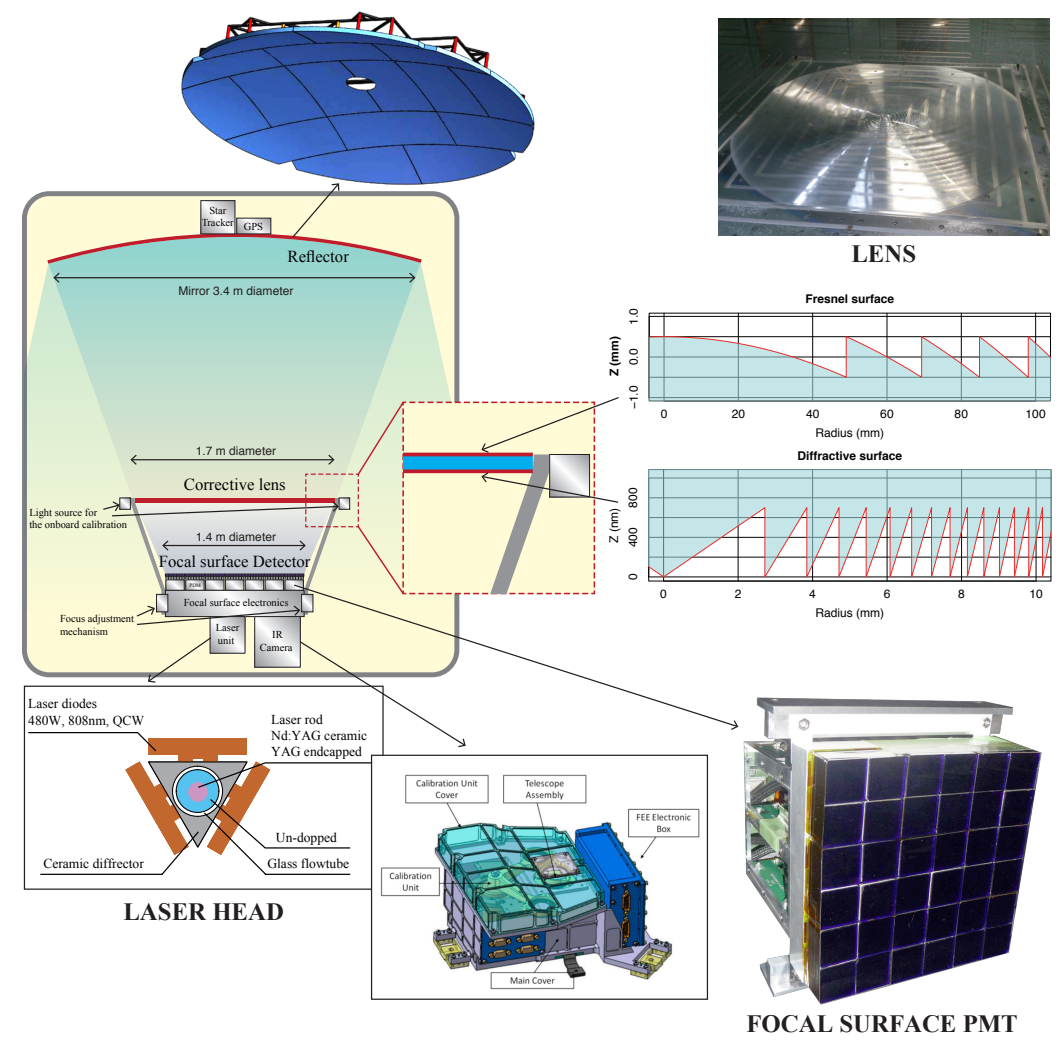

Figure 2: Conceptual scheme of the K-EUSO telescope. Light is collected by a mirror, focused by a Fresnel refractive-diffractive lens and detected by the Focal Surface (FS) electronics. Below the FS is located the atmospheric monitoring system. The items to be constructed in Japan are the lens, the FS photo-multipliers and the Lidar laser head.

\begin{tabular}{|l|l||l|l|}
\hline \multicolumn{2}{|c|}{ Parameters of the Instruments } & \multicolumn{2}{c|}{ Parameters of the Mission } \\
\hline Field of view & $\pm 14^{\circ}$ & Operational period & $3 \mathrm{yr}+3 \mathrm{yr}$ \\
\hline Observational area & $>3.1 \times 10^{4} \mathrm{~km}^{2}$ & launching Rocket & Soyuz \\
\hline Optical bandwidth & $300-400 \mathrm{~nm}$ & Transportation to ISS & Progress \\
\hline Focal surface area & $1.45 \mathrm{~m}^{2}$ & Module & MRM-1 \\
\hline Number of pixels & 119,808 & Mass & $500 \mathrm{~kg}$ \\
\hline Pixel size & $2.88 \times 2.88 \mathrm{~mm}$ & Power (operative) & $300 \mathrm{~W}$ \\
\hline Pixel field of view & $0.058^{\circ}$ & Power (non-operative) & $100 \mathrm{~W}$ \\
\hline Event time sampling & $2.5 \mu \mathrm{s}$ & Data transfer rate & $100 \mathrm{kbps}$ \\
\hline Duty cycle & $\sim 20 \%$ & Altitude & $\sim 400 \mathrm{~km}$ \\
\hline & & Orbital inclination & $51.64^{\circ}$ \\
& &
\end{tabular}

Table 1: K-EUSO Mission Parameters 


\begin{tabular}{|l|l|l|}
\hline & \multicolumn{1}{|c|}{ KLYPVE } & \multicolumn{1}{c|}{ K-EUSO } \\
\hline Optics type & Mirror & Mirror + Corrective lens \\
\hline FOV & $\pm 7.5^{\circ}$ & $\pm 14^{\circ}$ \\
\hline Mirror diameter & $3.6 \mathrm{~m}$ Aspherical & $3.4 \mathrm{~m}$ Aspherical \\
\hline Mirror area & $10 \mathrm{~m}^{2}$ & $9 \mathrm{~m}^{2}$ \\
\hline Focal distance & $3 \mathrm{~m}$ & $4 \mathrm{~m}$ \\
\hline Lens diameter & - & $1.7 \mathrm{~m}$ (Fresnel + Diff) \\
\hline FS diameter & $1.2 \mathrm{~m}$ & $1.4 \mathrm{~m}$ \\
\hline Number of pixels & 2,500 & 119,808 \\
\hline Pixel angular size & $0.311^{\circ}$ & $0.058^{\circ}$ \\
\hline Pixel FOV in atm. & $2143 \mathrm{~m}$ & $400 \mathrm{~m}$ \\
\hline Orbit height & $400 \mathrm{~km}$ & $400 \mathrm{~km}$ \\
\hline
\end{tabular}

Table 2: Characteristic comparison between KLYPVE and K-EUSO telescope

\section{The baseline optical system design for K-EUSO}

The baseline optical system design for K-EUSO is consistent of the primary mirror, the Fresnel lens and the focal surface. The $3.4 \mathrm{~m}$ primary mirror, of Russian construction, is the evolution of the TUS Mirror[6, 7]. The reflective surface, which is aspherical surface, will gather light from the EAS UV photons and then concentrate them on the Focal Surface. The use of the mirror enables to get a large photon collection efficiency. The design of the K-EUSO optics is shown in Fig. 3. The $1.7 \mathrm{~m}$ corrective Fresnel lens, of Japanese construction, increases the field of view and reduces aberrations. The K-EUSO has a low focal number of F\# 0.857 . The material of Fresnel lens is UV transparent PMMA (polymethyl methacrylate) $[9,10]$ allows the telescope to satisfy the constraints posed by space-borne experiments. Furthermore, the small thickness of the lens allows the mass of the optics to be reduced resulting in a light system capable of withstanding launch vibration and thermal environments. The primary mirror and the corrective lens will be segmented to fit the Progress cargo ship and mounted on the MRM-1 module. The $1.7 \mathrm{~m}$ corrective lens is a double sided curved Fresnel design. The base curvature of the lens is $18715.5 \mathrm{~mm}$. The thickness of the lens is $10 \mathrm{~mm}$. The expected mass of the lens without the support structure is $27.0 \mathrm{~kg}$ (Best effort). The front surface of the lens, (facing to the primary mirror) has the normal Fresnel grooves. The back surface, (facing to the focal surface) has precision diffractive grooves for color correction. The characteristics of both surface are shown in Table 3. The FS has a spherical shape and is 1.4 $\mathrm{m}$ in diameter. The radius of curvature of the FS is $15984.9 \mathrm{~mm}$.

\section{The performance of the K-EUSO optics}

The RMS spot size of the base line K-EUSO optical design is $3.1 \mathrm{~mm}-5.6 \mathrm{~mm}$ in diameter. The spot diagram is shown in Fig. 4. The photon collection efficiencies (PCE) are 50\%@0 field angle,38\%@7 field angle and 17\%@14 field angle if that the diffractive efficiency of the lens is $95 \%$ and the reflectivity of the primary mirror is $90 \%$. PCEs are calculated with a formula in our 


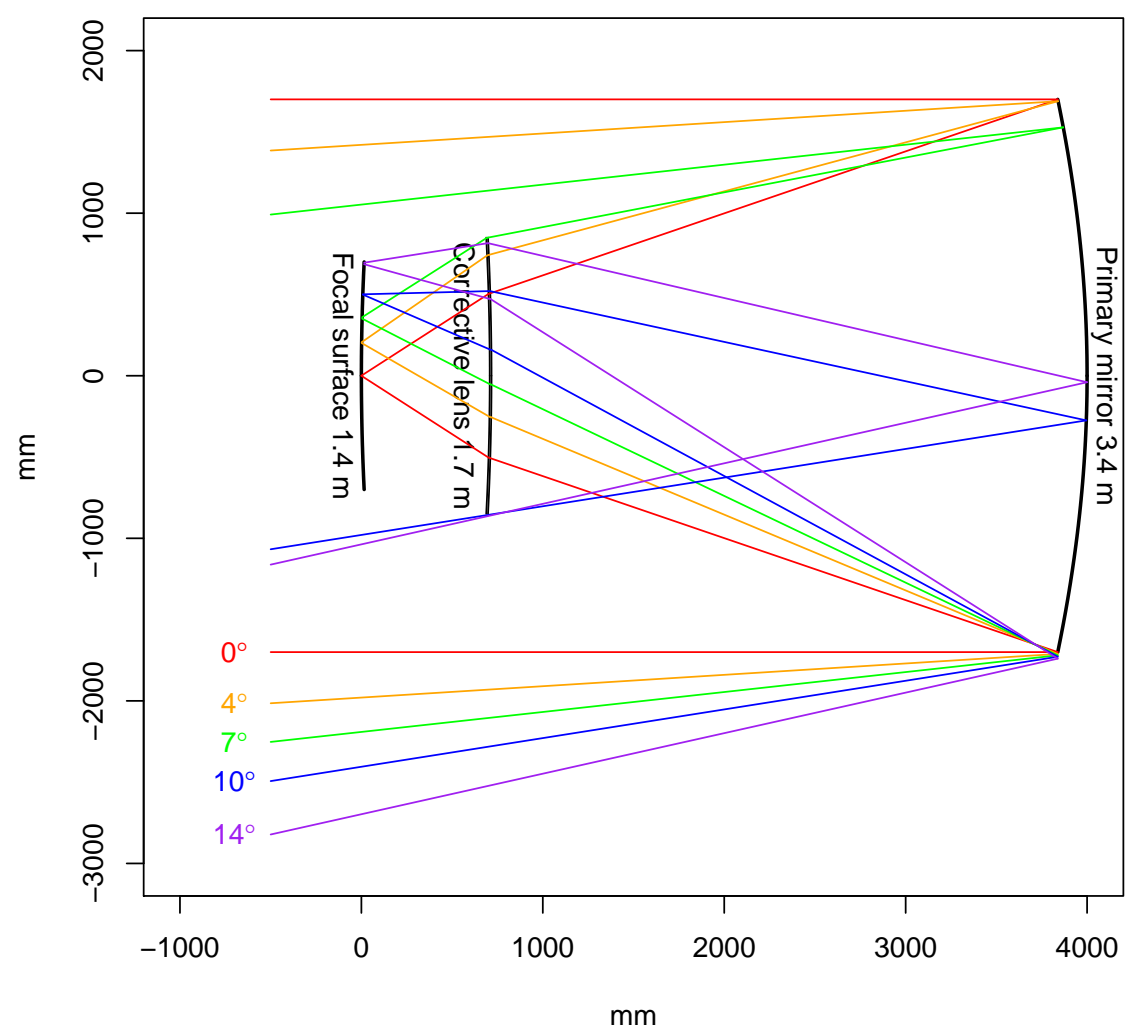

Figure 3: Optics design of the K-EUSO telescope. The $3.4 \mathrm{~m}$ diameter mirror (right) light from the EAS (coming from left). A $1.7 \mathrm{~m}$ diameter lens (center) corrects the coma aberration and increases the field of view allowing $\mathrm{a} \pm 14^{\circ}$ field of view on the $1.4 \mathrm{~m}$ Focal Surface (left).

\begin{tabular}{|l|l|l|}
\hline & \multicolumn{1}{|c|}{ The front surface } & The back surface \\
\hline Surface type & Normal Fresnel grooves & Precision grooves \\
\hline Groove edge height & $1.0 \mathrm{~mm}$ & $0.7 \mu \mathrm{m}$ \\
\hline Groove pitch & $0.82 \mathrm{~mm} \sim 54.11 \mathrm{~mm}$ & $5.3 \mu \mathrm{m} \sim 2.9 \mathrm{~mm}$ \\
\hline Number of Grooves & 694 & 78903 \\
\hline
\end{tabular}

Table 3: Characteristic of the correction Fresnel lens surfaces 

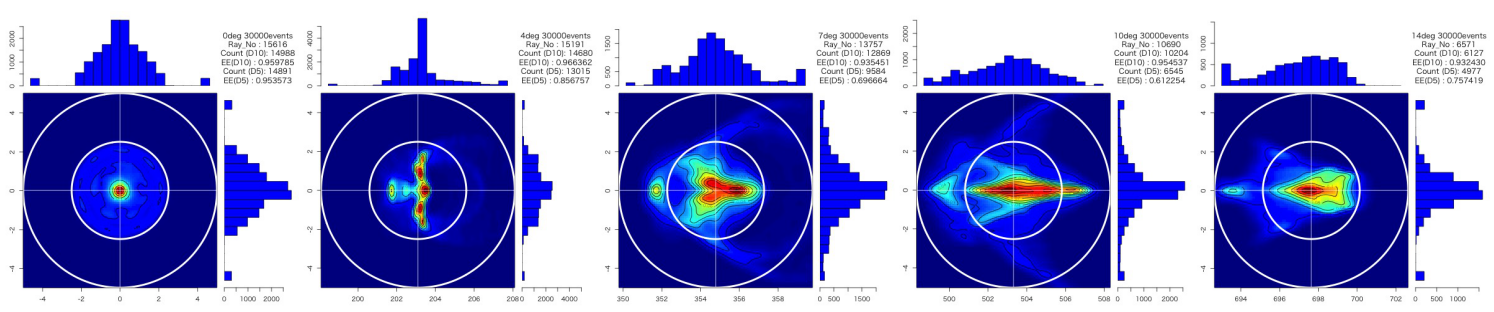

Figure 4: Point spread function of the optical system for light coming parallel with different inclination (from left to right, $0^{\circ}, 4^{\circ}, 7^{\circ}, 10^{\circ}$ and $14^{\circ}$ ). The diameter of the inner white circle is $5 \mathrm{~mm}$ and the outer circle is $10 \mathrm{~mm}$.

developed raytrace simulation:

$$
P C E=\frac{\text { Photon counts within a range of } 5 \mathrm{~mm} \text { diameter on the focal surface }}{\text { Photon counts which go to the primary mirror }}
$$

Photon counts which go to the primary mirror in the formula include those photons that are blocked by the focal detector and the lens. The plot of PCEs as function of field angles is shown in Fig. 5.

K-EUSO Baseline optics

(Diffractive efficiency: 95\%, Mirror reflectivity: 90\%)

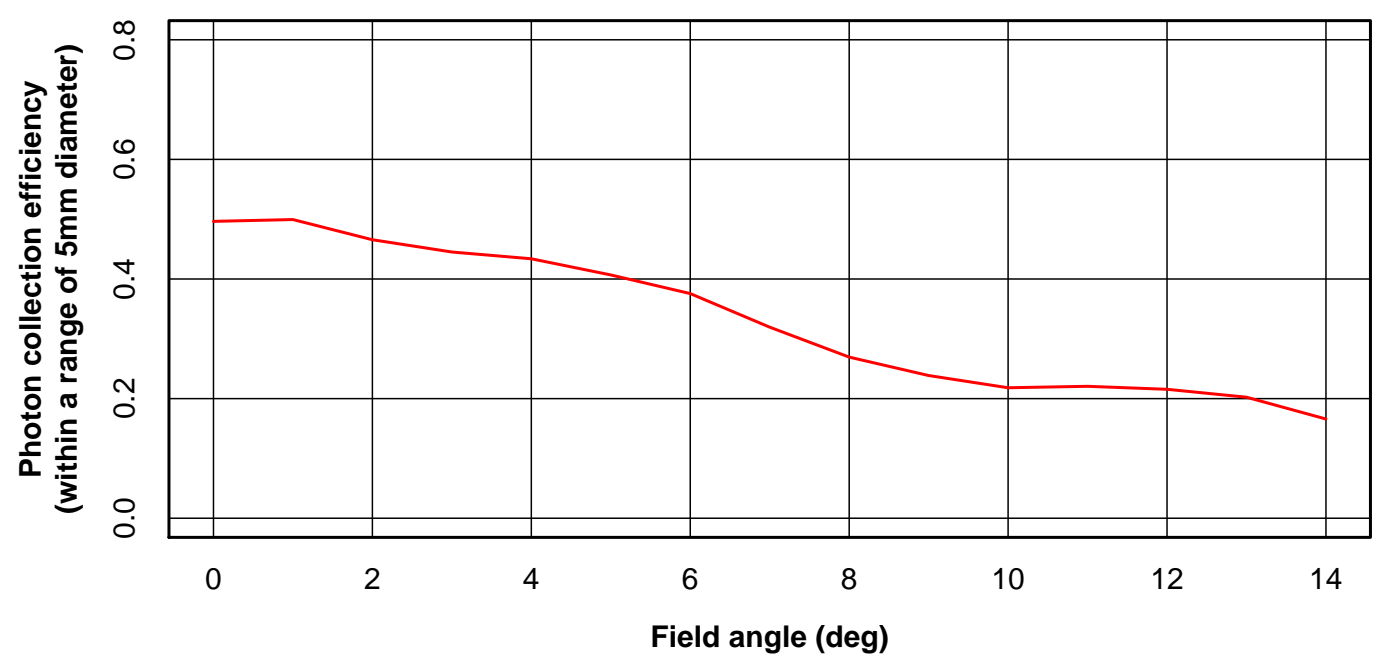

Figure 5: photon collection efficiencies of the optical system for light coming from showers of different inclination between $0^{\circ}$ and $14^{\circ}$ ).

Expected annual exposures of K-EUSO and the original KLYPVE are estimated by relative $\mathrm{S} / \mathrm{N}$ ratio from the JEM-EUSO annual exposure, which is calculated by using the Euso Simulation and Analysis Framework (ESAF). The expected annual exposure of K-EUSO on the whole celestial sphere is about 7 times of the original KLYPVE, about 2 times of Auger, about 10 times of Telescope Array and about 0.3 times of JEM-EUSO. Comparison of the expected annual exposure of KLYPVE, Auger, JEM-EUSO and K-EUSO is shown in Fig. 6. The details of K-EUSO performance are described in [11]. 


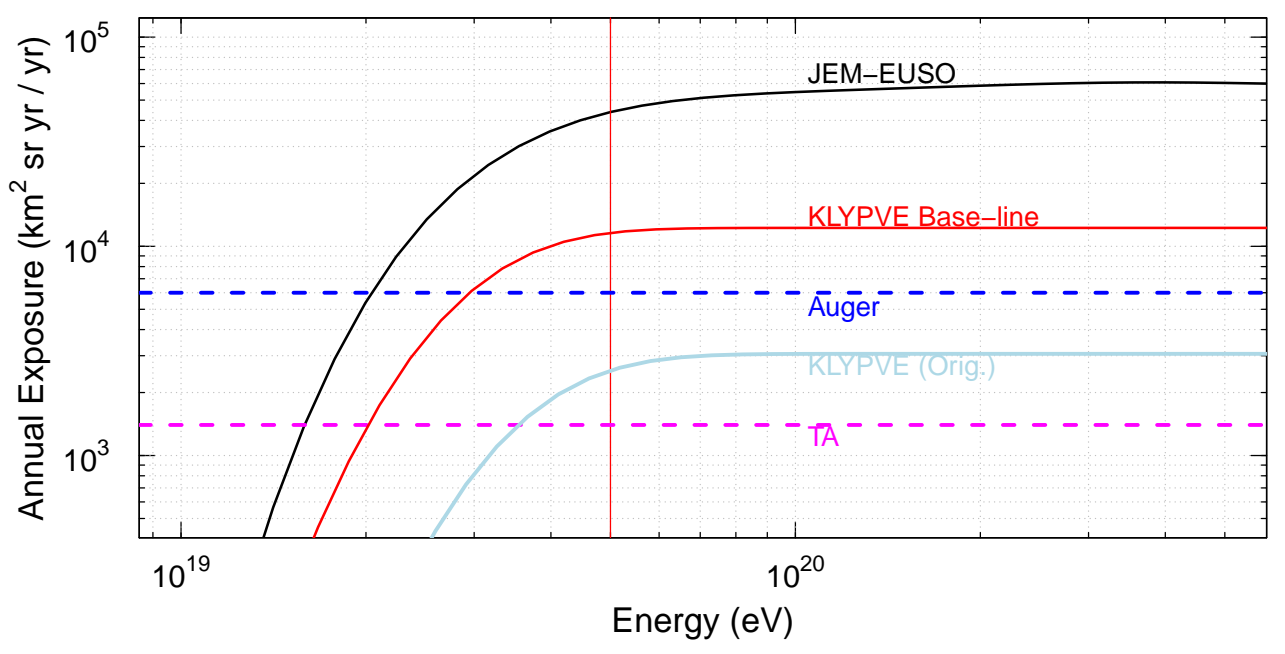

Figure 6: Annual exposure of K-EUSO as a function of energy compared with JEM-EUSO and the ground based Auger and Telescope Array observatories. The exposure of K-EUSO tends to be come horizontal around $5 \times 10^{19} \mathrm{eV}$ (Red vertical line). At lower energies, the efficiency decreases because of the lower number of UV photons generated in an UHECR shower.

\section{Conclusions}

K-EUSO enlarges the field of view to $\pm 14^{\circ}$ with reducing aberrations by using a corrective lens and improves the effective area by more than a factor 7 from the original KLYPVE. K-EUSO will achieve about twice the exposure of Auger per year above $5 \times 10^{19} \mathrm{eV}$ and can demonstrate the space-based observation by quantitative comparison between those obtained from the ground based observatories.

\section{References}

[1] R. U. Abbasi et al., The Astrophysical Journal Letters 790 (2014) 21

[2] The Pierre Auger Collaboration, Science 318 (2008) 938-943

[3] The Pierre Auger Collaboration, Astroparticle Physics 34 (2010) 314-326

[4] Garipov, GK et al., Experimental Techniques 49 (2006) 126-130

[5] Sadovnichy, VA et al., Solar System Research 45 (2011) 3-29

[6] Alexandrov, V et al. 27th ICRC, Hamburg, Germany, 2001

[7] M. Panasyuk et al. ECRS-2014 2014

[8] J.H. Adams Jr. et al. - JEM-EUSO Collaboration, Astroparticle Physics 44 (2013) 76

[9] EUSO collaboration, ESA-EUSO PhaseA study report (2004)

[10] Y. Takizawa et al. 33rd ICRC, RIO DE JANEIRO, 2013

[11] N. Sakaki et al. 34th ICRC, The Hague, The Netherlands, 2015 